\title{
INTERACCIONES ENTRE Fusarium verticillioides (Sacc.) Nirenberg, Aspergillus flavus Link Y Talaromyces funiculosus Thom EN AMBIENTES HERMÉTICOS
}

\section{INTERACTIONS BETWEEN Fusarium verticillioides (Sacc.) Nirenberg, Aspergillus flavus Link AND Talaromyces funiculosus Thom IN HERMETIC ENVIRONMENTS}

\author{
Claudia C. Castellari ${ }^{1 *}$, Facundo J. Marcos Valle ${ }^{1}$, y Ana M. Pacin ${ }^{2}$
}

\author{
${ }^{1}$ Facultad de Ciencias Agrarias, Universidad Nacional de Mar del Plata, Ruta Nacional 226 Km 73,5 \\ (B7620) Balcarce, Buenos Aires, Argentina. ORCID: https://orcid.org/0000-0001-6829-8374. F. M. Valle \\ ORCID: https://orcid.org/0000-0002-4077-900X \\ ${ }^{2}$ Fundación de Investigaciones Científicas Teresa Benedicta de la Cruz, M. Dorronzoro 141, Luján \\ (B6700FTA), Argentina. ORCID: https://orcid.org/0000-0002-3961-6588 \\ *Autor para correspondencia: E-mail: claudia_castellari@yahoo.com.ar; \\ ccastellari@mdp.edu.ar; castellari.claudia@inta.gob.ar
}

\section{RESUMEN}

El maíz es infectado naturalmente por especies fúngicas micotoxigénicas, como Fusarium verticillioides y Aspergillus flavus, y no micotoxigénicas, como Talaromyces funiculosus, durante el cultivo y en el almacenamiento convencional o hermético, como el silo bolsa. Se desconocen las interacciones entre estas especies y las estrategias de supervivencia en ambientes herméticos. El objetivo de este estudio fue determinar el efecto del ambiente hermético sobre las interacciones entre $F$. verticillioides, $A$. flavus y $T$. funiculosus. Se evaluaron, in vitro, $F$. verticillioides, $A$. flavus y T. funiculosus, obtenidos de granos de maíz almacenados en silos bolsa. Las especies crecieron confrontadas de a pares en placas de Petri, sin restricción de $\mathrm{O}_{2}$ y en microcultivos en ambientes herméticos con dos concentraciones iniciales de $\mathrm{O}_{2} \mathrm{y} \mathrm{CO}_{2}$. Se determinó la inhibición del crecimiento y las estrategias de colonización de cada especie utilizando microscopía estereoscópica y óptica. El ambiente hermético redujo el crecimiento y alteró la morfología de estructuras somáticas y reproductivas de las especies micotoxigénicas. T. funiculosus inhibió el crecimiento de ambas especies micotoxigénicas y produjo parasitismo de hifas. Se observó entremezcla mutua de las hifas entre las especies micotoxigénicas. Los niveles de $\mathrm{O}_{2}$ y de $\mathrm{CO}_{2}$ en los ambientes herméticos variaron en el tiempo, según el par de especies que creció confrontada en cada uno de ellos. El estudio de las interacciones fúngicas en ambientes herméticos aportó información valiosa para comprender la colonización y permanencia de especies productoras de micotoxinas asociadas a granos durante su almacenamiento en sistemas herméticos, como el silo bolsa.

Palabras clave: micobiota micotoxigénica, maíz, Zea mays, ambiente hermético, silo bolsa.

\section{ABSTRACT}

Corn is naturally infected by mycotoxigenic fungal species such as $F$. verticillioides, $A$. flavus and T. funiculosus. Interactions between the species and survival strategies under hermetic conditions are unknown. The objective of this study was to determine the effect of the hermetic environment on the interactions between $F$. verticillioides, A. flavus and T. funiculosus. F. verticillioides, A. flavus and 
T. funiculosus were evaluated in vitro. The species grew in pairs in Petri dishes without $\mathrm{O}_{2}$ restriction and in microcultures in hermetic environments with two initial concentrations of $\mathrm{O}_{2}$ and $\mathrm{CO}_{2}$. Growth inhibition and colonization strategies of each species were determined using stereoscopic and light microscopy. The hermetic environment reduced growth and altered the morphology of somatic and reproductive structures of the mycotoxigenic species. T. funiculosus inhibited the growth of both mycotoxigenic species and caused hyphal parasitism. Mutual intermingling of the hyphae was observed between the mycotoxigenic species. The levels of $\mathrm{O}_{2}$ and $\mathrm{CO}_{2}$ in the hermetic environments varied according to the pair of species that grew up in each of them. The study of fungal interactions in hermetic environments provided information to understand the colonization and permanence of mycotoxin producing species associated with grain during their storage in hermetic systems, such as the bag.

Key words: micotoxigenic mycobiota, maize, Zea mays, hermetic environment, bag silo.

\section{INTRODUCCIÓN}

El sistema de almacenaje denominado silo bolsa constituye una alternativa para los granos de cereales y oleaginosas. Las bolsas tienen un alto grado de hermeticidad a los gases $\left(\mathrm{O}_{2}\right.$ y $\left.\mathrm{CO}_{2}\right)$ por lo que el incremento del $\mathrm{CO}_{2}$ producto de la respiración reduce el desarrollo de los insectos y hongos evitando el deterioro de los granos (Bartosik, 2012).

El maíz es susceptible a la infección fúngica y a la contaminación con micotoxinas durante el cultivo y en el almacenamiento. Este evento es regido por factores extrínsecos, factores intrínsecos, y factores implícitos (Torres et al., 2003; Hammond et al., 2004; Marín et al., 2004; Sanchis et al., 2007; Giorni et al., 2009; Castellari, 2017).

Las especies micotoxigénicas aisladas de granos de maíz con mayor frecuencia pertenecen a los géneros Fusarium y Aspergillus (Gregori et al., 2013) mientras que las especies de Penicillium y Talaromyces son las que se aíslan con mayor frecuencia entre las poblaciones no micotoxigénicas (Castellari et al., 2015). Los resultados del análisis de micotoxinas en maíz revelaron que entre el 90 y $100 \%$ de las muestras están contaminadas con fumonisinas (FB) (Pacin et al., 2009) el 10\% con zearalenona y deoxinivalenol, y entre el 10 y $50 \%$ con aflatoxinas (AF) (Garrido et al., 2012).

De las especies de Fusarium, F. verticillioides productora de FB es aislada con mayor frecuencia en maíz cultivado en regiones templadas de Argentina y otros países (Marín et al., 2013). Diversos estudios evaluaron la ocurrencia de la especie en diferentes etapas del cultivo (Martínez et al., 2010; Picot et al., 2011), de la cosecha y el almacenamiento de los granos (Marcos Valle, 2015). En regiones agrícolas con temperaturas cálidas, el maíz es contaminado con mayor frecuencia por A. flavus, por lo que su ocurrencia es estudiada en la región centro y norte de la Argentina (Chulze et al., 1989; Pacin et al., 2001a; Pacin et al., 2001b; Mutti et al., 2009; González Pereyra et al., 2011) y en otros países con características climáticas similares (Sekiyama et al., 2005; García y Heredia, 2006; Giorni et al., 2007).

El estudio de las relaciones entre las especies fúngicas asociadas a distintas matrices, donde no existen limitaciones en el intercambio gaseoso, reveló diferentes estrategias de competencia (Benhamound y Brodeur, 2000; Baig et al., 2012; Quiroz-Sarmiento et al., 2008; Leggieri et al., 2919). Sin embargo, las interacciones entre especies micotoxigénicas en ambientes herméticos, como en los silos bolsa que almacenan granos, no fue estudiada y se cree que niveles de $\mathrm{O}_{2}$ menores a 5\% afectarían las estrategias de competencia y colonización. El objetivo del trabajo fue estudiar las interacciones entre F. verticillioides, A. flavus y T. funiculosus, in vitro, en ambientes herméticos con diferente composición inicial de $\mathrm{O}_{2}$ y $\mathrm{CO}_{2}$, y determinar cambios en el crecimiento $\mathrm{y}$ alteraciones en las estructuras somáticas y reproductivas que afecten la colonización y la persistencia.

\section{MATERIALES Y MÉTODOS}

\section{Aislamientos fúngicos}

Se evaluaron 18 aislamientos de F. verticillioides, A. flavus y T. funiculosus, seis de cada especie, obtenidos de granos de maíz almacenados en silos bolsa, ubicados en tres localidades (Balcarce, General Alvarado y Tandil) de la zona sudeste

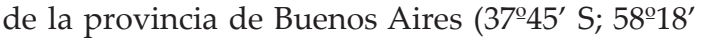
W; $130 \mathrm{msnm})$, Argentina. Las muestras de granos de maíz se tomaron en dos tiempos del almacenamiento: al inicio (T1: 10 días) y al final (T2: 150 días), coincidiendo con las estaciones de invierno (año 2010) y verano (año 2011). La identificación se realizó empleando la taxonomía convencional (Nelson et al., 1983; Summerell et al., 2003; Pitt y Hocking, 2009) y la molecular para confirmar F. verticillioides y A. flavus (Cheverri y Samuels, 2003). 
En ambos tiempos de muestreo se registraron las concentraciones de $\mathrm{O}_{2}$ y CO$~_{2}$ en las bolsas (in situ) con un medidor de gases portátil (Check Point, PBI, DanSensor, Dinamarca) y el pH de los granos de las muestras, en el laboratorio con un peachímetro digital y portátil (Oakton modelo PH11, $N^{\circ}$ serie 203852), según protocolo de Castellari et al. (2015).

\section{Obtención de inóculos}

Cada aislamiento fúngico fue sembrado en placas de Petri de $90 \mathrm{~mm}$ de diámetro con medio agar papa glucosa (2\%) (APG) (Britania ${ }^{\circledR}$ ) al que se le adicionó cloranfenicol (Sigma ${ }^{\circledR}$ ) al 0,02\%. Las placas se incubaron siete días a $25^{\circ} \mathrm{C}$, en oscuridad. El micelio y los conidios de cada placa fueron extraídos con $2 \mathrm{~mL}$ de una solución de glicerol $\left(\right.$ Biopak $\left.^{\circledR}\right)$ estéril $(35 \% \mathrm{v} / \mathrm{v})$, colocados en tubos eppendorf de 1,5 mL y conservados en freezer a $-20^{\circ} \mathrm{C}$. Los aislamientos están depositados y disponibles en el cepario del Laboratorio de Microbiología de Suelos y Alimentos de la Unidad Integrada Balcarce (EEA INTA Balcarce-Facultad de Ciencias Agrarias, Universidad Nacional de Mar del Plata (UNMdP), Argentina).

\section{Determinación de la tasa de crecimiento}

El inóculo de cada aislamiento fue ajustado en $10^{6}$ propágulos $\mathrm{mL}^{-1}$, en solución de $\mathrm{NaCl}(0,8 \%)$ y $3 \mu \mathrm{L}$ del stock se sembró en cuatro placas de Petri conteniendo medio APG $\left(\right.$ Britania $^{\circledR}$ ) con dos niveles de $\mathrm{pH}: 6,5$ ( $\mathrm{pH}$ de los granos en T1) y 5,6 ( $\mathrm{pH}$ de los granos en T2). Las placas se incubaron sin restricción de $\mathrm{O}_{2}$ a $25^{\circ} \mathrm{C}$ y a partir del tercer día y durante siete días se registró el diámetro de las colonias $(\mathrm{mm})$. Se midieron dos diámetros de cada colonia, se obtuvo el promedio de cada una y por especie $\mathrm{y}$, se calculó la tasa de crecimiento $\left(\mathrm{mm} \mathrm{día}{ }^{-1}\right)$.

Los datos fueron analizados empleando el paquete Rcmder (R Core Team, 2012). Se realizó un análisis de la varianza (ANOVA) considerando el efecto del $\mathrm{pH}$ sobre el diámetro de las colonias. Las diferencias entre las medias se determinaron con el test de comparaciones múltiples del paquete agricolae (Tukey, 5\%) (R Core Team, 2012).

\section{Detección de la inhibición del crecimiento confrontado entre $A$. flavus, $F$. verticillioides y T. funiculosus}

Todos los aislamientos fueron inoculados de a pares en los extremos opuestos de placas de Petri con medio APG $\left(\right.$ Britania $\left.^{\circledR}\right)$ ajustado con dos niveles de $\mathrm{pH}$ : 6,5 y 5,6. Cada par de aislamientos fue evaluado por cuadruplicado. Paralelamente, cada aislamiento fue sembrado individualmente en el mismo medio de cultivo con el objeto de medir el crecimiento en ausencia del antagonista.
Las placas se incubaron a $25^{\circ} \mathrm{C}$ en oscuridad, durante siete días, o hasta que el micelio del aislamiento que creció solo cubrió por completo la superficie del medio de cultivo. A los siete días se midió el radio de la colonia de cada uno de los aislamientos, creciendo confrontados y solos. Se calculó el promedio de la medición de tres radios para cada aislamiento y para cada especie $(\mathrm{mm} \pm \mathrm{SD})$, por cada nivel de $\mathrm{pH}$. Se determinó el porcentaje de inhibición del crecimiento de ambas especies confrontadas, respecto de cada una de ellas creciendo sola.

Los datos se analizaron por medio de un ANOVA y se determinó el efecto del crecimiento confrontado y del $\mathrm{pH}$ del medio de cultivo sobre el radio de crecimiento de la colonia.

Las interacciones entre los pares de aislamientos se caracterizaron macroscópicamente utilizando un microscopio estereoscópico (ZEISS 47526, $\mathrm{f}=100$, Alemania) y se determinó el tipo de interacción de acuerdo con Magan y Lacey (1984).

\section{Ambiente hermético de crecimiento confrontado} entre $A$. flavus, F. verticillioides y T. funiculosus

El efecto del ambiente hermético sobre las interacciones entre las hifas de las tres especies se evaluó con la técnica de microcultivos de Paul (1999) modificada, ya que no se utilizó formaldehído para fijar las estructuras $y$, en su reemplazo, se retiró el cubreobjeto, dejando secar el portaobjeto en cercanía de la llama de un mechero. Para describir las interacciones se utilizó la escala de Magan y Lacey (1984). Paralelamente, se caracterizó en el tiempo la composición de gases del ambiente hermético en el interior de frascos (de 1,550 $\mathrm{cm}^{3}$ de capacidad) donde crecieron confrontadas las tres especies. Las composiciones iniciales de gases en el interior de los frascos fueron: T1: $21 \%$ de $\mathrm{O}_{2}$ y $0 \%$ de $\mathrm{CO}_{2}$; y T2: $5 \%$ de $\mathrm{O}_{2}$ y $15 \% \mathrm{CO}_{2}$ (las concentraciones correspondieron a las registradas en las bolsas herméticas que almacenaron maíz en T1 y T2, respectivamente). Se confeccionaron cuatro frascos por cada composición de gases. La mezcla 2 fue adquirida a la Empresa Air Liquide Argentina S.A., en cilindros de aluminio de $1 \mathrm{~L}$ (volumen de agua) con balance nitrógeno. El regulador utilizado fue un regulador Alphagaz, cuerpo inoxidable y presión de salida de 0 a 8 bar. Para el llenado de los frascos, una vez colocadas las placas con los microcultivos se utilizó la técnica de vacío: bomba de vacío DVL 150, de vacío máximo $0,030 \mathrm{~mm} \mathrm{Hg}$; desplazamiento 50 $\mathrm{Hz}-150 \mathrm{~L} \mathrm{~min}^{-1}$. La presión de trabajo de vacío fue de $68 \mathrm{~mm} \mathrm{Hg} \mathrm{min}^{-1}$ y el tiempo para lograr el vacío fue de $2 \mathrm{~min}$. La presión de trabajo de llenado con la mezcla fue de $5 \mathrm{~L} \mathrm{~min}^{-1}$, logrando el llenado con 4 min de trabajo, aproximadamente. 
Para lograr la hermeticidad del frasco luego del llenado se utilizó sellador universal de silicona $3 \mathrm{M}^{\circledast}$ en el septo, colocado en la tapa. Se registró diariamente la evolución de la concentración de $\mathrm{O}_{2}$ y $\mathrm{CO}_{2}$ con un medidor de gases portátil (Check Point, PBI, DanSensor, Dinamarca). Las mediciones finalizaron cuando se registraron tres lecturas consecutivas diarias de $0 \%$ de $\mathrm{O}_{2}$. Los pares de datos se presentaron en gráficos que permitieron observar la evolución diaria de las concentraciones de $\mathrm{O}_{2}$ y CO 2 conforme avanzó el crecimiento confrontado de las especies.

\section{RESULTADOS Y DISCUSIÓN}

Caracterización del crecimiento de A. flavus, $F$. verticillioides y T. funiculosus en ambiente sin restricción de $\mathrm{O}_{2}$

Los diámetros de las colonias de A. flavus a los siete días de incubación fueron de 73,9 $( \pm 4,60)$ $\mathrm{mm}$ y 77,3 $( \pm 3,40) \mathrm{mm}$ para niveles de $\mathrm{pH}$ de 5,6 y 6,5, respectivamente. Se detectó un efecto del tiempo $(\mathrm{P}<0,001)$ y $\mathrm{pH}(\mathrm{P}<0,001)$, sobre el diámetro de las colonias, el que se incrementó diariamente y en el medio de cultivo con menor acidez $(\mathrm{pH}$ 6,5). Las velocidades promedio de crecimiento observadas para esta especie fueron de 4,41 mm día ${ }^{-1}$ y $4,58 \mathrm{~mm}$ día $^{-1}$, para los niveles de $\mathrm{pH} 5,6$ y 6,5, respectivamente.

La velocidad de crecimiento de $F$. verticillioides fue menor que la registrada para $A$. flavus. Los diámetros de las colonias a los siete días de incubación fueron de 53,4 $( \pm 10,07) \mathrm{mm}$ y $71,1( \pm 10,36) \mathrm{mm}$ para niveles de $\mathrm{pH}$ de $5,6 \mathrm{y}$ 6,5 , respectivamente. Se detectó un efecto $(\mathrm{P}$ $=0,0117)$ de interacción entre $\mathrm{pH}$ y tiempo. A partir del tercer día de incubación los diámetros de las colonias desarrolladas en $\mathrm{pH} 6,5$ se incrementaron significativamente $(\mathrm{P}<0,05)$. No se observó incremento significativo $(\mathrm{P}>0,05)$ de los diámetros, para ambos niveles de $\mathrm{pH}$ entre el sexto y séptimo día de incubación. Las velocidades de crecimiento promedio fueron de 2,28 $\mathrm{mm}$ día $^{-1}$ y de 3,2 $\mathrm{mm}_{\text {día }}{ }^{-1}$ para los $\mathrm{pH}$ de 5,6 y 6,5 , respectivamente.

T. funiculosus no fue afectada $(\mathrm{P}>0,05)$ por el $\mathrm{pH}$ del medio de cultivo para crecer en condiciones sin restricción de $\mathrm{O}_{2}$. Sólo se observó un efecto del tiempo de incubación $(\mathrm{P}<0,001)$ sobre el diámetro de las colonias. Para ambos niveles de $\mathrm{pH}$, los diámetros se incrementaron significativamente $(P<0,05)$ entre el primer $y$ séptimo día, desde el inicio de las mediciones. Las velocidades de crecimiento para T. funiculosus fueron de $3,60 \mathrm{~mm}$ día $^{-1}$ y 3,48 $\mathrm{mm}_{\text {día }}{ }^{-1}$ para los niveles de $\mathrm{pH}$ de 5,6 y 6,5, respectivamente.

Antagonismo entre A. flavus, $F$. verticillioides y T. funiculosus en ambientes sin restricción de $\mathrm{O}_{2}$

La reducción de los diámetros de las colonias se observó cuando las especies crecieron confrontadas. A. flavus ejerció un efecto inhibitorio mayor sobre $F$. verticillioides, que éste sobre $A$. flavus (Tabla 1). El $\mathrm{pH}$ del medio de cultivo no afectó $(\mathrm{P}>0,05)$ el desarrollo de ambas especies creciendo en confrontación $(A$. flavus: $\mathrm{P}=0,455 ;$ F. verticillioides: $\mathrm{P}=0,8722$ ). Para el caso de $A$. flavus creciendo con $F$. verticillioides, las hifas presentaron una interacción de tipo A (mezcla de hifas en el encuentro) de acuerdo con la clasificación de Magan y Lacey (1984).

El pH no afectó $(\mathrm{P}>0,05)$ el crecimiento de T. funiculosus en cultivo puro; sin embargo, este factor sí afectó el crecimiento de las colonias de $T$.

Tabla 1. Inhibición de A. flavus y $F$. verticillioides creciendo en confrontación en ambiente sin restricción de $\mathrm{O}_{2}$.

Table 1. Inhibition of A. flavus and F. verticillioides in confrontation, in an environment without $\mathrm{O}_{2}$ restriction.

\begin{tabular}{lcc}
\hline \multicolumn{1}{c}{ Especie } & $\begin{array}{c}\text { Radio de la colonia } \\
(\mathbf{m m} \pm \text { SD)* }\end{array}$ & $\begin{array}{c}\text { Inhibición del } \\
\text { crecimiento (\%) }\end{array}$ \\
\hline A. flavus & $80,00( \pm 0,00)$ & \\
Testigo - pH 5,6 & $80,00( \pm 0,00)$ & \\
Testigo - pH 6,5 & $37,33( \pm 2,51)$ & 53,33 \\
Creciendo con F. verticillioides (pH 5,6) & $40,00( \pm 5,00)$ & 50,00 \\
Creciendo con F. verticillioides (pH 6,5) & & \\
F. verticillioides & $76,00( \pm 0,00)$ & \\
Testigo - pH 5,6 & $78,00( \pm 0,00)$ & \\
Testigo - pH 6,5 & $28,33( \pm 2,88)$ & 62,76 \\
Creciendo con A. flavus (pH 5,6) & $28,00( \pm 1,73)$ & 64,00 \\
Creciendo con A. flavus (pH 6,5) & \\
\hline
\end{tabular}

*Promedio de 6 aislamientos y 4 repeticiones. 
funiculosus $(\mathrm{P}=0,018)$ y también de $F$. verticillioides $(\mathrm{P}=0,0036)$ creciendo en confrontación. Los mayores radios de crecimiento para $T$. funiculosus se observaron en el medio con $\mathrm{pH}$ 5,6, mientras que para $F$. verticillioides los mayores radios de crecimiento se registraron en el medio con $\mathrm{pH}$ 6,5 (Tabla 2).

T. funiculosus, en presencia de F. verticillioides, presentó una esporulación que cubrió rápidamente la superficie de placa de crecimiento a los tres días de incubación, e inhibió el crecimiento de $F$. verticillioides.

Al examinar los aislamientos de $A$. flavus creciendo en confrontación con los de $T$. funiculosus, se observó que los primeros fueron limitados en su crecimiento, ya que la superficie de la placa fue cubierta con microcolonias de $T$. funiculosus originadas por una rápida esporulación. Este evento, influyó negativamente $(\mathrm{P}<0,05)$ en el desarrollo de $A$. flavus, respecto de los mismos aislamientos creciendo en cultivo puro (Tabla 3).

Los resultados indican que $A$. flavus y $F$. verticillioides presentaron un comportamiento de crecimiento similar, caracterizado por la extensión de sus hifas y escasa esporulación. Una situación diferente fue observada para $T$. funiculosus, que inhibió el crecimiento de ambas especies micotoxigénicas, utilizando la estrategia de rápida esporulación de la superficie. La

Tabla 2. Inhibición del crecimiento de F. verticillioides y T. funiculosus creciendo en confrontación en ambiente sin restricción de $\mathrm{O}_{2}$.

Table 2. Growth inhibition of F. verticillioides and T. funiculosus in confrontation, in an environment without $\mathrm{O}_{2}$ restriction.

\begin{tabular}{lcc}
\hline \multicolumn{1}{c}{ Especie } & $\begin{array}{c}\text { Radio de la colonia } \\
(\mathbf{m m} \pm \text { SD)* }\end{array}$ & $\begin{array}{c}\text { Inhibición del } \\
\text { crecimiento (\%) }\end{array}$ \\
\hline T. funiculosus & $30,54( \pm 1,49)$ & \\
Testigo - pH 5,6 & $30,54( \pm 3,04)$ & \\
Testigo - pH 6,5 & $7,00( \pm 0,00)$ & 77,09 \\
Creciendo con F. verticillioides (pH 5,6) & $7,00( \pm 0,00)$ & 77,09 \\
Creciendo con F. verticillioides $\mathrm{pH}(6,5)$ & & \\
F. verticillioides & $27,66( \pm 4,41)$ & \\
Testigo - pH 5,6 & $35,77( \pm 8,40)$ & \\
Testigo - pH 6,5 & $10,75( \pm 3,16)$ & 61,13 \\
Creciendo con T. funiculosus (pH 5,6) & $14,05( \pm 3,56)$ & 60,74 \\
Creciendo con T. funiculosus (pH 6,5) & \\
\hline
\end{tabular}

${ }^{*}$ Promedio de 6 aislamientos y 4 repeticiones.

Tabla 3. Inhibición del crecimiento de las especies A. flavus y T. funiculosus creciendo en confrontación, en ambiente sin restricción de $\mathrm{O}_{2}$.

Table 3. Inhibition growth of $A$. flavus and T. funiculosus in confrontation, in an environment without $\mathrm{O}_{2}$ restriction.

\begin{tabular}{lcc}
\hline \multicolumn{1}{c}{ Especie } & $\begin{array}{c}\text { Radio de la colonia } \\
(\mathbf{m m} \pm \text { SD) }\end{array}$ & $\begin{array}{c}\text { Inhibición del } \\
\text { crecimiento (\%) }\end{array}$ \\
\hline T. funiculosus & $30,54( \pm 1,49)$ & \\
Testigo - pH 5,6 & $30,54( \pm 3,04)$ & \\
Testigo - pH 6,5 & $7,50( \pm 1,04)$ & 72,72 \\
Creciendo con A. flavus (pH 5,6) & $7,33( \pm 1,23)$ & 73,05 \\
Creciendo con A. flavus (pH 6,5) & & \\
A. flavus & $37,86( \pm 1,04)$ & \\
Testigo - pH 5,6 & $39,30( \pm 3,80)$ & 67,30 \\
Testigo - pH 6,5 & $12,38( \pm 2,32)$ & 59,44 \\
Creciendo con T. funiculosus (pH 5,6) & $15,94( \pm 1,83)$ & \\
Creciendo con T. funiculosus (pH 6,5) &
\end{tabular}

*Promedio de 6 aislamientos y 4 repeticiones. 
dispersión exagerada de las esporas en tiempo y espacio puede estar relacionada con una estrategia de competencia, de acuerdo con Wyatt et al. (2013).

La interacción de las hifas de cada una de las especies micotoxigénicas (A. flavus y $F$. verticillioides) creciendo en confrontación con $T$. funiculosus fue descripta como una inhibición por contacto tipo B, de acuerdo con la escala de Magan y Lacey (1984). Además, en la zona de contacto entre las hifas se observó una coloración roja intensa ocasionada posiblemente por la síntesis de un extrolito de T. funiculosus del cual no se tiene información detallada de su estructura química ni tampoco de su función (Yilmaz et al., 2014).

Características microscópicas de la interacción entre $A$. flavus, F. verticillioides y T. funiculosus en ambiente hermético

Cuando las cepas de A. flavus y F. verticillioides crecieron confrontadas con $T$. funiculosus en el ambiente T1 y sus hifas tomaron contacto, se observaron dos eventos relevantes. El primero fue la presencia de hifas y conidios teñidos de color rojo por T. funiculosus y su posterior lisis; el segundo fue la formación de apresorios por un aislamiento de T. funiculosus en las hifas de ambas especies micotoxigénicas, evidenciando una estrategia de parasitismo.

Cuando el contenido de $\mathrm{O}_{2}$ en el ambiente $\mathrm{T} 2$ se redujo a $5 \%$ y se incrementó la concentración de $\mathrm{CO}_{2}$, ambas especies micotoxigénicas mostraron sus hifas con engrosamiento, vacuolización, estructuras globosas y una mayor ramificación en la zona de contacto con T. funiculosus. En coincidencia con la rápida reducción del $\mathrm{O}_{2}$ del ambiente $\mathrm{T} 2$, todos los aislamientos de $T$. funiculosus produjeron apresorios en las hifas de A. flavus.

Cuando se estudió microscópicamente la interacción entre las hifas, el hallazgo de alteraciones morfológicas en Aspergillus coincidieron con otras descripciones (QuirozSarmiento et al., 2008). Las hifas de F. verticillioides y A. flavus presentaron una ramificación exagerada pudiendo constituir una estrategia para colonizar rápidamente el espacio. En cambio, T. funiculosus produjo apresorios y lisis celular en A. flavus y F. verticillioides, lo que también podría ser considerado como estrategia de adaptación y supervivencia de esta especie.

Composición de $\mathrm{O}_{2}$ y $\mathrm{CO}_{2}$ en ambientes herméticos durante el crecimiento confrontado

En el ambiente T1, a los siete días de incubación de las cepas de $F$. verticillioides y $A$. flavus creciendo confrontadas, no se registró $\mathrm{O}_{2}$ y la concentración de $\mathrm{CO}_{2}$ fue de 16,95\%. En el ambiente $\mathrm{T} 2$, el $\mathrm{O}_{2}$ se consumió completamente en cinco días, mientras que el $\mathrm{CO}_{2}$ se incrementó a valores del 18\% (Fig. 1).

La Fig. 2 muestra la evolución de los niveles de gases cuando $F$. verticillioides y $T$. funiculosus crecieron en confrontación. En el ambiente T1 no se detectó $\mathrm{O}_{2}$ a los seis días de incubación y el $\mathrm{CO}_{2}$ se incrementó a valores de $17,50 \%$, en ese tiempo. En el ambiente $\mathrm{T} 2 \mathrm{el}_{2}$ se redujo a valores inferiores a $1 \%$ luego de 23 días de incubación, tiempo en el que el $\mathrm{CO}_{2}$ se incrementó a niveles
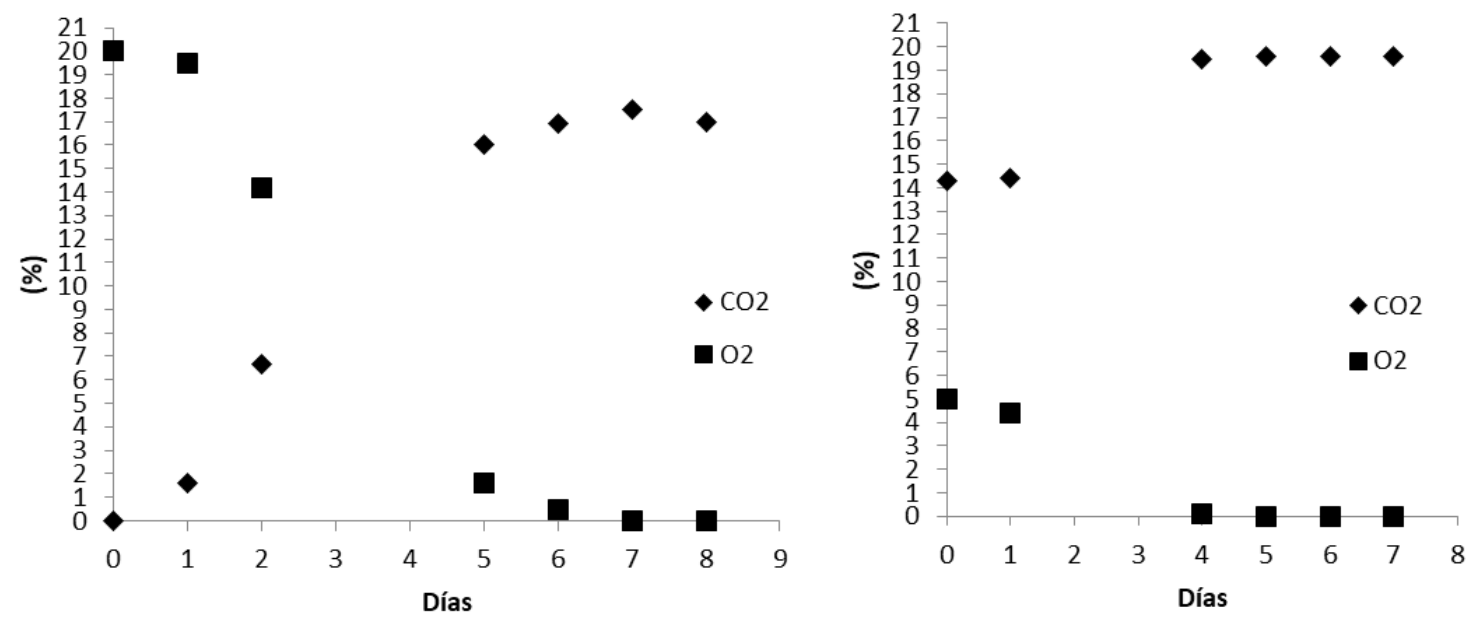

Fig. 1. Evolución de las concentraciones (\%) de $\mathrm{O}_{2}$ y $\mathrm{CO}_{2}$ en los ambientes T1 (izquierda) y T2 (derecha), donde crecieron $F$. verticillioides y A. flavus en confrontación.

Fig. 1. Evolution of $\mathrm{O}_{2}$ and $\mathrm{CO}_{2}$ concentrations (\%) in environments T1 (left) and T2 (right), $F$. verticillioides and $A$. flavus in confrontation. 
que superaron el $18 \%$.

Cuando $A$. flavus y $T$. funiculosus crecieron en confrontación en el ambiente T1, se detectó $\mathrm{O}_{2}$ hasta el día 24 de incubación, mientras que en el ambiente T2 no se detectó $\mathrm{O}_{2}$ en el sistema a los seis días de iniciado el ensayo (Fig. 3).

El estudio de la evolución de las concentraciones de $\mathrm{O}_{2}$ y $\mathrm{CO}_{2}$ de los ambientes herméticos donde crecieron confrontadas y de a pares las tres especies, mostró que el nivel de $\mathrm{CO}_{2}$ inicial en el ambiente hermético influyó en el consumo de $\mathrm{O}_{2}$ de $A$. flavus y $F$. verticillioides y como consecuencia, en su tasa de crecimiento.
Castellari et al. (2014) determinaron que la tasa de crecimiento de $F$. verticillioides en el ambiente T1 fue de $1,59 \mathrm{~mm} \mathrm{~d}^{-1}$, un $30 \%$ inferior a la registrada en condiciones sin restricción de $\mathrm{O}_{2}$, y la tasa de crecimiento de $A$. flavus en el mismo ambiente fue de $2,11 \mathrm{~mm} \mathrm{~d}^{-1}$, un $52 \%$ menor a la registrada en condiciones de cultivo con intercambio de $\mathrm{O}_{2}$.

F. verticillioides fue la especie que consumió en menor tiempo el $\mathrm{O}_{2}$ disponible en el ambiente hermético T1, cuando creció con A. flavus y $T$. funiculosus, influenciado posiblemente por la ausencia de $\mathrm{CO}_{2}$. Por otro lado, de las Figs. 1, 2 y 3 se deduce que $A$. flavus fue responsable de la
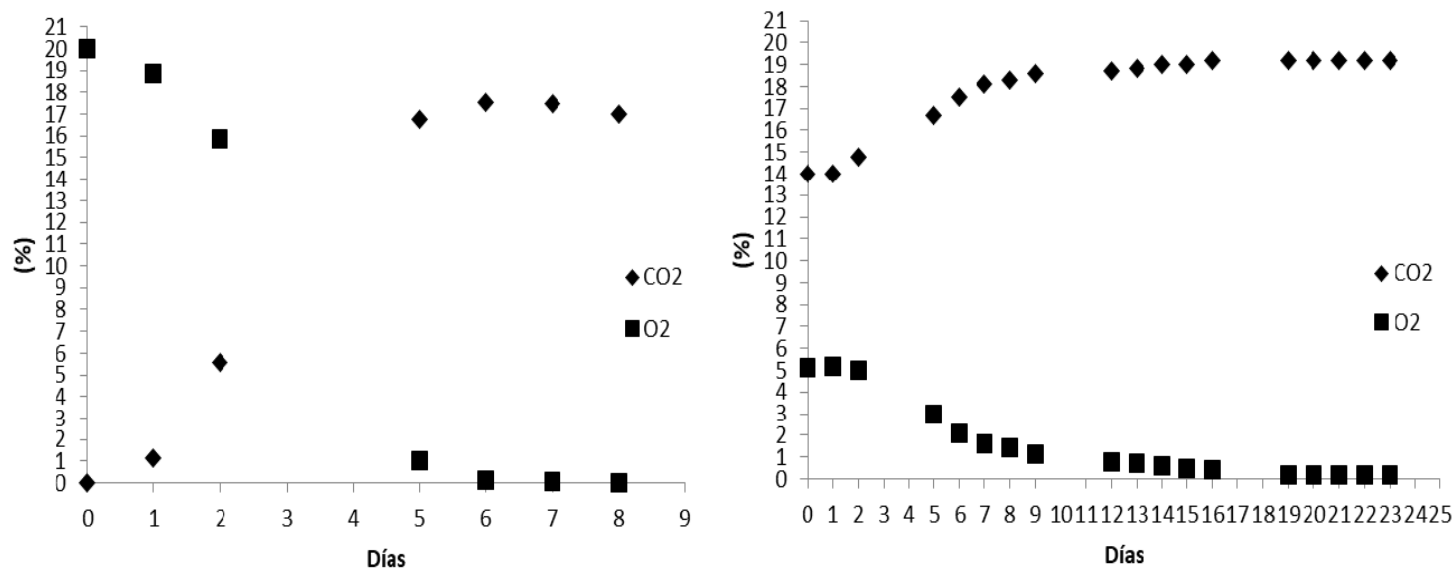

Fig. 2. Evolución de las concentraciones (\%) de $\mathrm{O}_{2}$ y $\mathrm{CO}_{2}$ en los ambientes T1 (izquierda) y T2 (derecha), donde crecieron $F$. verticillioides y $T$. funiculosus en confrontación.

Fig. 2. Evolution of $\mathrm{O}_{2}$ and $\mathrm{CO}_{2}$ concentrations (\%) in environments $\mathrm{T} 1$ (left) and T2 (right), in $F$. verticillioides and $T$. funiculosus in confrontation.
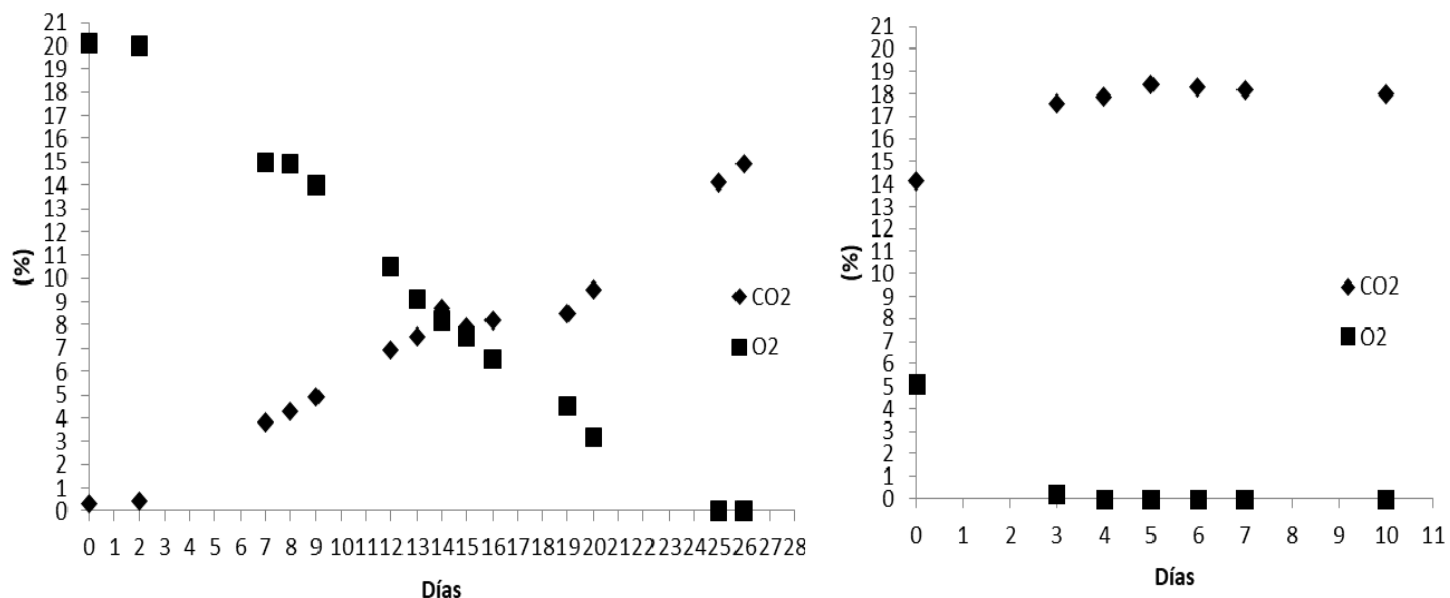

Fig. 3. Evolución de las concentraciones (\%) de $\mathrm{O}_{2} \mathrm{y} \mathrm{CO}_{2}$ en los ambientes T1 (izquierda) y T2 (derecha), donde crecieron A. flavus y $T$. funiculosus en confrontación.

Fig. 3. Evolution of $\mathrm{O}_{2}$ and $\mathrm{CO}_{2}$ concentrations (\%) in environments $\mathrm{T} 1$ (left) and T2 (right), in $A$. flavus and T. funiculosus in confrontation. 
reducción del $\mathrm{O}_{2}$, en el ambiente con un nivel inicial de 5\% (T2), cuando creció con F. verticillioides y T. funiculosus, infiriendo que el nivel de $\mathrm{CO}_{2}$ superior a $15 \%$ afectaría negativamente la tasa de crecimiento de estas especies. Las tres especies modificaron en forma diferente los niveles de $\mathrm{O}_{2}$ y $\mathrm{CO}_{2}$ de cada uno de los ambientes herméticos durante su crecimiento, evidenciado por un consumo distinto de $\mathrm{O}_{2}$ en función del tiempo.

\section{CONCLUSIONES}

El crecimiento de las especies micotoxigénicas F. verticillioides y A. flavus y de T. funiculosus (no micotoxigénica) fue afectado por el ambiente hermético y por la presencia de otras especies. El ambiente hermético alteró la morfología de estructuras somáticas y reproductivas asexuales de las especies micotoxigénicas. T. funiculosus inhibió el crecimiento de ambas especies micotoxigénicas a través de una esporulación rápida de sus colonias. Asimismo, el número de aislamientos de esta especie con actividad parasítica se incrementó cuando la concentración inicial de $\mathrm{O}_{2}$ en el ambiente hermético fue menor a 5\%. La evolución de las concentraciones de $\mathrm{O}_{2}$ y $\mathrm{CO}_{2}$ en ambientes de crecimiento hermético varió según las especies fúngicas presentes.

Este trabajo proporcionó evidencia que indica que evaluar la composición fúngica micotoxigénica y no micotoxigénica asociada a granos almacenados en ambientes herméticos, constituye un factor implícito de relevancia a considerar cuando se pretenden planificar estrategias para evitar el deterioro de los granos y la producción de micotoxinas durante el tiempo en que éstos están acopiados.

\section{RECONOCIMIENTOS}

El presente trabajo fue financiado por la Universidad Nacional de Mar del Plata por los proyectos "Factores implícitos determinantes del establecimiento de la micobiota micotoxigénica en granos de maíz almacenados en atmósferas automodificadas" (Código AGR 441/14) y "Factores ambientales que afectan los patrones de uso de nutrientes y las relaciones entre Fusarium verticillioides (Sacc.) Nirenberg, Aspergillus flavus Link y Talaromyces funiculosus (syn. P. funiculosum Thom), aislados de maíz almacenado en silo bolsas en Argentina" (Código AGR 499/16).

Los autores agradecen al grupo de Poscosecha de granos de la Unidad Integrada Balcarce, Dr. Ricardo Bartosik e Ing. Agr. Leandro Cardoso y al Sr. Martín Mansilla del Laboratorio de Microbiología de Suelos y Alimentos, por la asistencia técnica.

\section{LITERATURA CITADA}

Bartosik, R. 2012. An inside look at the silo-bag system. p. 117-128. In Navarro S., Banks H.J., Jayas D.S., Bell C.H., Noyes R.T., and Ferizli A.G. (eds.). Proc. 9th. Int. Conf. on Controlled Atmosphere and Fumigation in Stored Products. CAF. 15-19 de Octubre. Antalya, Turkey. ARBER Professional Congress Services, Turkey.

Baig, M., F. Sumia, V.B. Kadam, and Y. Shaikh. 2012. Utilization of antagonist against seed borne fungi. Trends in Life Sciences 1:42-46.

Benhamou, N., and J. Brodeur. 2000. Evidence for antibiosis and induced host defense reactions in the interaction between Verticillium lecanii and Penicillium digitatum. The causal agent of green mold. Phytopathology 90:932-943.

Castellari, C.C. 2017. Factores determinantes en el desarrollo de poblaciones fúngicas micotoxigénicas en granos de maíz almacenado en bolsas plásticas herméticas. Tesis doctoral. 406 p. Doctorado en Ciencias Agrarias. Universidad Nacional de Mar del Plata, Argentina.

Castellari, C.C., M.G. Cendoya, F. Marcos Valle, V. Barrera, y A.M. Pacin. 2015. Factores extrínsecos e intrínsecos asociados a poblaciones fúngicas micotoxigénicas de granos de maíz (Zea mays L.) almacenados en silos bolsa en Argentina. Revista Argentina de Microbiología 47:350-359.

Castellari, C.C., F.J. Marcos Valle, M.O. Mansilla y A.M. Pacin. 2014. Influencia del ambiente hermético sobre el crecimiento y esporulación de poblaciones fúngicas asociadas a granos de maíz almacenados en silobolsa en la provincia de Buenos Aires, Argentina. p. 50-51. En Primer Congreso Internacional de Silo bolsa. 13-16 de Octubre. Mar del Plata, Buenos Aires, Argentina. INTA, Buenos Aires, Argentina. ISBN 978-987-33-6146-3.

Chaverri, P., and G.J. Samuels. 2003. Hypocreal Trichoderma (Ascomycota, Hypocreales, Hypocreaceae): Species with green ascospores. Studies in Mycology 48:1-116.

Chulze, S., C. Bertinetti, A. Dalcero, M. Etcheverry, C. Farnochi, A. Torres, et al. 1989. Incidence of aflatoxin, zearalenone, and deoxynivalenol on corn in Argentina. Mycotoxin Research 5:9-12.

García, S., and N. Heredia. 2006. Mycotoxins in Mexico: epidemiology, management, and control strategies. Mycopathologia 162:255264.

Garrido, C.E., C. Hernández Pezzani, and A.M. Pacin. 2012. Micotoxin occurrence in Argentina's maize (Zea mays L.) from 1999 to 2010. Food Control 25:660-665. 
Giorni, P., N. Magan, A. Pietri, T. Bertuzzi, and P. Battilani. 2007. Studies on Aspergillus Section Flavi isolated from maize in northern Italy. International Journal of Food Microbiology 113:330-338.

Giorni, P., N. Magan, and P. Battilani. 2009. Environmental factors modify carbon nutritional patterns and niche overlap between Aspergillus flavus and Fusarium verticillioides strains from maize. International Journal of Food Microbiology 130:213-218.

Gregori, R., P. Meriggi, A. Pietri, S. Formenti, and G. Baccarini. 2013. Dynamics of fungi and related mycotoxins during cereal storage in silo bags. Food Control 30:280-287.

González Pereyra, M.L., S.M. Chiacchiera, C. Rosa, R. Sager, A.M. Dalcero, and L. Cavaglieri. 2011. Comparative analysis of the mycobiota and mycotoxins contaminating corn trench silos and silo bags. Journal of the Science Food and Agriculture 91:1474-1481.

Hammond, B.G., K.W. Campbell, C.D. Pilcher, T.A. Degoyer, A.E. Robinson, B.L. Mcmillen, et al. 2004. Lower fumonisin mycotoxin levels in the grain of Bt corn grown in the United State in 2000-2002. Journal of Agricultural and Food Chemistry 52:1390-1397.

Leggieri, L.C., P. Giorni, A. Pietri and P. Battilani. 2019. Aspergillus flavus and Fusarium verticillioides Interaction: Modeling the impact on mycotoxin production. Frontiers in Microbiology 10:1-10.

Magan, N., and J. Lacey. 1984. Effect of water activity, temperature and substrate on interactions between field and storage fungi. Transaction of the British Mycological Society 82:83-93.

Marín, S., A.J. Ramos, G. Cano-Sancho, and V. Sanchis. 2013. Mycotoxins: ocurrence, toxicology, and exposure assessment. Food Chemistry Toxicology 60:218-237.

Marín, S., N. Magan, A. Ramos, and V. Sanchis. 2004. Fumonisin-producing strains of Fusarium: A review of their ecophysiology. Journal of Food Protection 67:1792-1805.

Martínez, M., R. Moschini, D. Barreto, J. Bodega, R. Comerio, H. Forjan, et al. 2010. Factores ambientales que afectan el contenido de fumonisina en granos de maíz. Tropical Plant Pathology 35:277-284.

Marcos Valle, F.J. 2015. Tasa respiratoria de granos de maíz (Zea mays) y su microbiota asociada en almacenamiento hermético. 153 p. Tesis Magister Scientiae. Universidad Nacional de Mar del Plata, Balcarce, Argentina.
Mutti, J., C. Castellari, F. Marcos Valle, L. Cardoso, y R. Bartosik. 2009. Micobiota toxigénica en granos de maíz almacenados en bolsas plásticas herméticas. En XII Congreso de Ciencia y Tecnología de los Alimentos. 9-12 de Octubre. Concordia, Entre Ríos, Argentina. Asociación Argentina de Tecnólogos Alimentarios, AATA. Ciudad Autónoma de Buenos Aires, Argentina.

Nelson, P.E., T.A. Toussoun, and W.F.O. Marasas. 1983. Fusarium species. An illustrated manual of identification. 193 p. The Pennsylvania State University Press, University Park, Pennsylvania, USA.

Paul, B. 1999. Suppression of Botrytis cinerea causing the grey mould disease of grapevine by an aggressive mycoparasite, Pythium radiosum. FEMS Microbiology Letters 176:2530.

Pacin, A.M., L.E. Broggi, S.L. Resnik, and H.H.L. González. 2001a. Mycoflora and mycotoxin natural occurrence in corn from Entre Rios Province, Argentina. Mycotoxin Research 17:31-38.

Pacin, A.M., S.L. Resnik, and M.M. Samar. 2001b. Micotoxinas en el maíz argentino en el período: 1995-2000. p. 7-9. En $7^{\circ}$ Congreso de Maíz. 7-9 de noviembre de 2001. Pergamino, Buenos Aires, Argentina. Centro de Investigaciones en Micotoxinas (CIM), Universidad Nacional de Luján, Buenos Aires, Argentina.

Pacin, A.M., E. Ciancio Bovier, H.H.L. González, E.M. Whitechurch, E.J. Martínez, and S.L. Resnik. 2009. Fungal and fumonisins contamination in Argentine maize (Zea mays L.) silo bags. Journal of Agricultural and Food Chemistry 57:2778-2781.

Picot, A., C. Barreau, L. Pinson-Gadais, F. Piraux, L. Caron, C. Lannou, et al. 2011. The dent stage of maize kernels is the most conducive for fumonisin biosynthesis under field conditions. Applied Environmental Microbiology 77 (23):8382-8390.

Pitt, J., and A.D. Hocking. 2009. Fungi and food spoilage. 524 p. Blackie Academic \& Professional, London, UK.

Quiroz-Sarmiento, V.F., R. Ferrera Cerrato, A. Alarcón, y M.E. Lara Hernández. 2008. Antagonismo in vitro de cepas de Aspergillus y Trichoderma hacia hongos filamentosos que afectan al cultivo de ajo. Revista Mexicana de Micología 26:27-34.

R Core Team. 2012. R: A language and environment for statistical computing. $\mathrm{R}$ Foundation for Statistical Computing, Vienna, Austria. Available at http://www.Rproject.org/ (Accessed 01 November 2017). 
Sanchis, V., S. Marín, and A.J. Ramos Girona. 2007. Factores determinantes en la producción de micotoxinas. p. 64-89. En M. Soriano del Castillo (ed.). Micotoxinas en alimentos. Días de Santo, Madrid, España.

Summerell, B.A., B. Salleh, and J.F. Leslie. 2003. A utilitarian approach to Fusarium identification. Plant Disease 87:117-128.

Sekiyama, B.L., A.B. Ribeiro, P.A. Machinski, and M.Jr. Machinski. 2005. Aflatoxins, ochratoxin and zearalenone in maize-based food products. Brazilian Journal of Microbiology 36:289-294.

Torres, M.R., A.J. Ramos, J. Soler, V. Sanchis, and S. Marín. 2003. SEM study of water and temperature effects on the initial growth of Aspergillus ochraceus, Alternaria alternata and Fusarium verticillioides on maize grain. International Journal of Food Microbiology 81:184-193.
Yilmaz, N., C.M. Visagie, J. Houbraken, J.C. Frisvad, and R.A. Samson. 2014. Polyphasic taxonomy of the genus Talaromyces. Studies Mycology 78:175-41.

Wyatt, T.T., H.A.B. Wösten, and J. Dijksterhuis. 2013. Chapter two: fungal spores for dispersion in space and time. Advances in Applied Microbiology 85:43-91. 